\title{
Récits et cadrages politiques en Euskadi : lectures de l'iconographie abertzale
}

Tales and political framing in Euskadi: an analysis of the abertzale iconography

\section{Xavier Crettiez}

\section{(2) OpenEdition}

Journals

Édition électronique

URL : http://journals.openedition.org/conflits/18791

DOI : 10.4000/conflits. 18791

ISSN : $1777-5345$

Éditeur :

CECLS - Centre d'études sur les conflits - Liberté et sécurité, L'Harmattan

Édition imprimée

Date de publication : 31 décembre 2013

Pagination : 81-100

ISBN : 978-2-343-02618-3

ISSN : 1157-996X

Référence électronique

Xavier Crettiez, « Récits et cadrages politiques en Euskadi : lectures de l'iconographie abertzale »,

Cultures \& Conflits [En ligne], 91/92 | automne/hiver 2013, mis en ligne le 31 décembre 2014, consulté le 31 mars 2021. URL : http://journals.openedition.org/conflits/18791 ; DOI : https://doi.org/10.4000/ conflits. 18791 


\section{Récits et cadrages politiques en Euskadi : lectures de l'iconographie abertzale}

\section{Xavier CRETTIEZ}

Xavier Crettiez est Professeur de science politique à l'université de VersaillesSaint-Quentin-en-Yvelines et chercheur au CESDIP. Spécialiste des violences ethno-nationalistes et de la sociologie de la mobilisation, il a récemment codirigé avec Isabelle Sommier, Les dimensions émotionnelles du politique, Rennes, PUR, 2012 et a coécrit avec Pierre Piazza, Murs rebelles, Paris, Karthala, 2013. Deux articles récents complètent cette étude sur la sociologie de la violence : "High Risk Activism : Essai sur le processus de radicalisation violente ", Pôle sud, $n^{\circ} 34,2011$ et $n^{\circ} 35,2011$.

U ne traversée du Pays basque offre assez rapidement à voir les marques de l'existence d'un conflit politique au cœur même d'un paysage d'une douceur peu commune. Ce ne sont pas tant les signes d'une possible militarisation de l'espace public - de moins en moins évidente à partir du début des années 2000 - qui troublent l'observateur, que les inscriptions nombreuses et difficilement compréhensibles qui ornent les murets routiers et les façades des immeubles. Graffitis, affiches, pochoirs, dessins viennent rappeler, dans une langue faiblement répandue qui renforce le sentiment d'emprise communautaire de l'espace public, la force d'une politique informelle dans cette région à cheval sur deux États puissants. Par politique informelle, on entendra avec Michel Offerlé et Laurent le Gall, des

« formes, des pratiques, des activités, des expressions qui, faute de bénéficier d'une reconnaissance et d'une légitimité de la part des prescripteurs et des agents les plus influents du champ, sont rejetées en dehors de ce champ quand bien même elles participent pleinement ou accessoirement à sa constitution ${ }^{1}$. »

1. Offerlé M. et Le Gall L., "La politique informelle entre incertitudes et inconstances » in Offerlé M., Le Gall L. et Ploux F. (eds.), La politique sans en avoir l'air, Rennes, PUR, 2012, p. 16. 
L'iconographie abertzale ${ }^{2}$ en Euskadi relève de la politique informelle, de cette «culture du pauvre » ou de cette « pratique opportuniste » qui, à rebours des usages politiques par le haut, développe une emprise politique de l'espace par le bas et surtout par les marges, rendant visibles des thématiques diverses, essentiellement identitaires, antirépressives et politiquement alternatives, largement ignorées par les acteurs institutionnels dominants ${ }^{3}$. Loin de participer à une «invisibilisation du politique " 4, l'iconographie prétend à l'inverse survisibiliser des enjeux locaux autant qu'elle met en scène des récits politiques variés.

C'est cette scénographie anonyme que l'on présentera dans cet article. Objet d'un relevé systématique dans la zone française du Pays basque et plus éparse dans la zone sud, espagnole, ce travail s'appuie donc sur un recueil comptable de plus d'un millier de traces iconographiques nationalistes dont on présentera le bilan dans une première partie. La couverture routière de la zone nord d'Euskadi a été de $64 \%$ des $611 \mathrm{~km}$ de routes nationales et départementales soit un peu plus de 390 kilomètres, incluant les principaux axes routiers susceptibles d'être couverts de traces iconographiques 5 . La deuxième partie de l'article cherchera à comprendre le cadrage opéré par les acteurs du nationalisme radical ainsi que les dessins instrumentaux d'une entreprise iconographique qui est aussi une véritable machine de production d'affects collectifs.

\section{L’iconographie abertzale : un état des lieux}

Le territoire basque sur lequel repose cette étude est binational et, à ce titre, présente un visage varié, fortement dépendant du cadre étatique dans lequel la contestation opère, rappelant à l'observateur que les formes comme les finalités de la protestation nationalitaire sont partiellement dictées par leur ancrage stato-national ${ }^{6}$. La partie espagnole de l'Euskadi-le Pays Basque sud

2. Abertzale signifie patriote révolutionnaire en euskera et se réfère habituellement à la frange radicale du nationalisme basque, soutien de l'organisation Euskadi Ta Askatasuna (ETA).

3. Précisons ici que cette "pratique informelle et sauvage » peut fort bien être le fait d'acteurs organisés à l'image des nationalistes radicaux qui se situent aux marges du champ politique et cultivent une pratique activiste reposant sur la défiance et le conflit. Cette étude bute évidemment sur l'identité précise des acteurs de ces graffs, impossibles à connaître vu la pratique clandestine privilégiée, même si on peut raisonnablement penser qu'elle soit le fait de la mouvance abertzale, sous initiative individuelle (les tags) ou plus institutionnelle (les stickers ou affiches), compte tenu des messages émis.

4. Offerlé M. et Le Gall L., op. cit., p. 30.

5. L'auteur voudrait remercier ici Pierre Piazza, compagnon indispensable sur les routes corses et basques, traquant les marques iconographiques ou acceptant de prendre le volant pendant de longues heures. Cette étude n'aurait pu advenir sans son amitié et son aide précieuse. Merci également à Fabien Jobard, directeur du CESDIP, qui a accepté le financement de cette mission.

6. Comme le rappelait opportunément Pierre Birnbaum dans La logique de l'État (Paris, Fayard, 1981). Sur un modèle quelque peu différent, Marc Steinberg souligne également que les pratiques et discours contestataires sont souvent prisonniers des cadres de perception dominants, ceux de l'État et de ses institutions structurantes : Steinberg M., Figthing Words: 
- centre névralgique de la contestation abertzale 7 , offre le visage d'une expression picturale de la contestation principalement axée sur des référents mémoriels et identitaires ainsi que sur l'affirmation d'un soutien continu au nationalisme guerrier et belliqueux incarné par l'organisation paramilitaire basque : ETA ${ }^{8}$. On présentera rapidement la situation iconographique au sud qui n'a pas fait l'objet d'un traitement empirique rigoureux avant de centrer notre présentation sur un état des lieux de la contestation murale au Nord.

Jusqu'au début de l'année 2010, six principaux outils d'affirmation iconographique sont massivement utilisés en Euskadi sud, allant du graffiti sauvagement inscrit sur les murs de la rue à la banderole, souvent accrochée aux balcons des quartiers populaires de Donostia ou Bilbao ou aux bords de routes, au pochoir, sur les murs des villes en passant par le dessin (ou fresque), par le sticker, apposé principalement sur les équipements publics et enfin par l'affiche ornant tout type de support. Trois registres dominants de messages émergent.

Le premier, minoritaire, relève de l'identitarisme, principalement axé autour de la représentation des contours du territoire basque auquel a été retirée la ligne de frontière politique et juridique, ne laissant subsister qu'une forme territoriale unique et unitaire. Cette naturalisation picturale d'un espace basque distinct, arraché à son emprise géographique des cartes d'Espagne et de France, favorise l'intériorisation du discours politique indépendantiste ( $c f$. document 1). L'ajout presque systématique de termes en appelant à l'indépendance, la liberté, l'amnistie et l'usage de l'euskera (la langue basque) renforce cette prétention - visuellement instituée - à l'altérité. L'appel à recourir à l'euskera est récurrent au sud, renforçant la résistance à la colonisation de l'espace public ${ }^{9}$ par la culture castillane, dominante et majoritairement rejetée ${ }^{10}$.

Working Class Formation, Collective Action and Discourse in Early Nineteen-Century England, Cornell University Press, 1999.

7. Le nationalisme basque émerge à la fin du XIXe siècle dans les provinces de Biscaye et du Guipozcoa et connait un revival militant sous le franquisme autour de l'organisation Euskadi Ta Askatasuna (ETA) à partir de 1959, se pérennisant sous la démocratie et débordant à la fin des années 1970 du côté français du Pays Basque. Sur le nationalisme basque on se reportera à Letamendia F., Historia del nacionalismo vasco y de ETA (trois tomes), Usurbil, RyB ediciones, 1994 et, en français, Elorza A. et al., ETA. Une histoire, Paris, Denoël, 2002.

8. Les observations concernant l'Euskadi sud proviennent d'un travail réalisé en 2010 par Sarah Bedrani et proposé dans le cadre d'un mémoire de master 2, intitulé La contestation politique des abertzals an Pays Basque espagnol: l'iconographie murale comme répertoire d'action (sous la direction de Xavier Crettiez), Université de Versailles Saint Quentin en Yvelines, juin 2010. Merci à Sarah Bedrani pour les informations qu'elle a bien voulu me communiquer.

9. Pour reprendre les termes de Jürgen Habermas pour qui les mouvements sociaux opèrent comme autant de tentatives de refus de la «colonisation du monde vécu » par un État dominant dans ses valeurs comme dans ses pratiques. Sur la notion de « colonisation du monde vécu » chez Habermas, voir Habermas J., Théorie de l'agir communicationnel, tome 2, Paris, Fayard, 1987, p. 404.

10. Si l'on en croit les tous derniers résultats électoraux donnant une large victoire aux formations nationalistes tenant un discours largement anti-espagnol. 


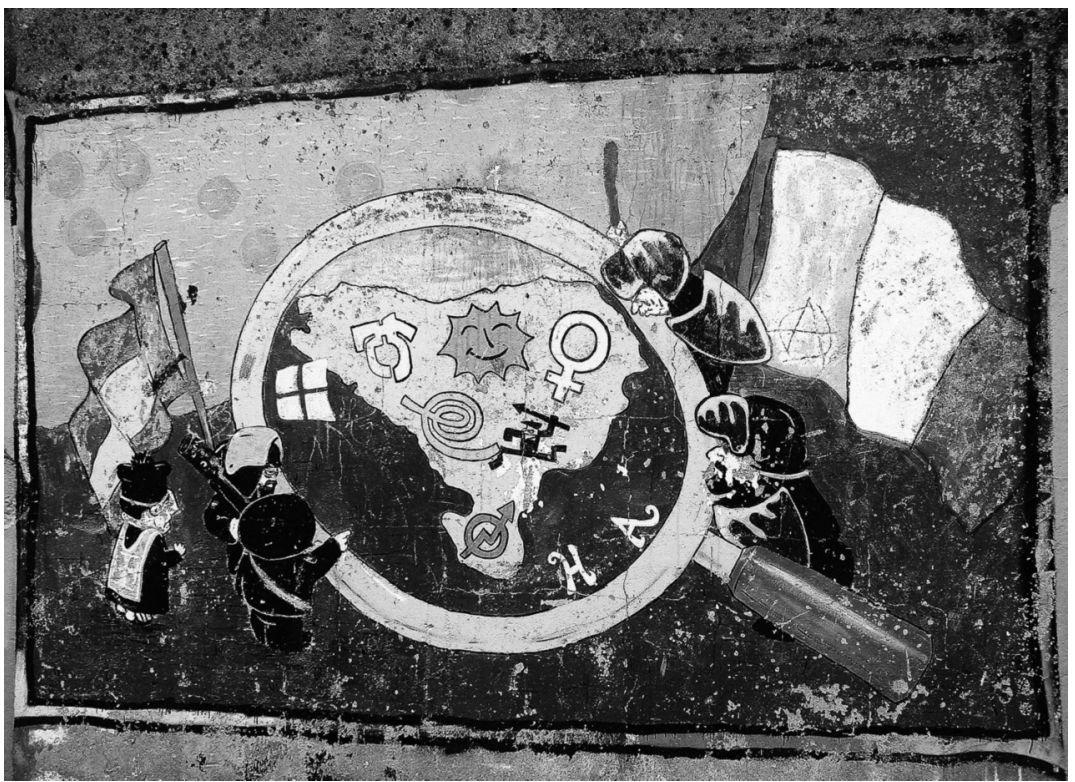

Document 1 - Fresque de Bayonne illustrant, au nord également, ce découpage schématique des contours du Pays basque

Le second registre, nettement plus affirmé, rassemble les signes d'une mémoire conflictuelle avec l'Espagne allant du rappel des conflits passés, et singulièrement de la guerre civile opposant un Pays Basque républicain à une Espagne franquiste, aux actes de torture et de mauvais traitements attribués aux forces de l'ordre espagnoles à l'encontre des militants nationalistes basques ( $c f$. document 2) ${ }^{11}$. L'usage sur de nombreux supports du tableau de Picasso, Guernica, illustrant le calvaire de cette ville symbole du Pays Basque soumise au bombardement des troupes allemandes en soutien à l'Espagne phalangiste, comme le rappel des tortures dans les commissariats pendant la période de transition ou l'enlèvement supposé de militants basques ${ }^{12}$, contribuent au maintien d'une culture d'opposition et à la pérennisation d'une «communauté de la peur »13, refusant tout compromis avec l'ennemi vu comme un éternel bourreau.

11. Sur cet usage instrumental d'un passé conflictuel, voir Muro D., "Nationalism and nostalgia: the case of radical Basque nationalism", Nations and Nationalism, 11-4, 2005, pp. 571 et s.

12. L'affaire Ion Anza, militant supposé d'ETA disparu en 2010 alimentera une mise en accusation partout visible sur les murs du Pays Basque, des services de police espagnols, accusés d'avoir enlevé puis tué un militant, qui plus est malade et fragile.

13. Crettiez X., Violence et nationalisme, Paris, Odile Jacob, 2006. Sur cette importance de la peur dans les dynamiques nationalitaires, voir Robin C., La peur. Histoire d'une idée politique, Paris, Amand Colin, 2006 et dans le cas espagnol, Baby S., "Sortir de la guerre civile à retardement : le cas espagnol », Histoire politique. Politique, culture, société, 3, 2007. 


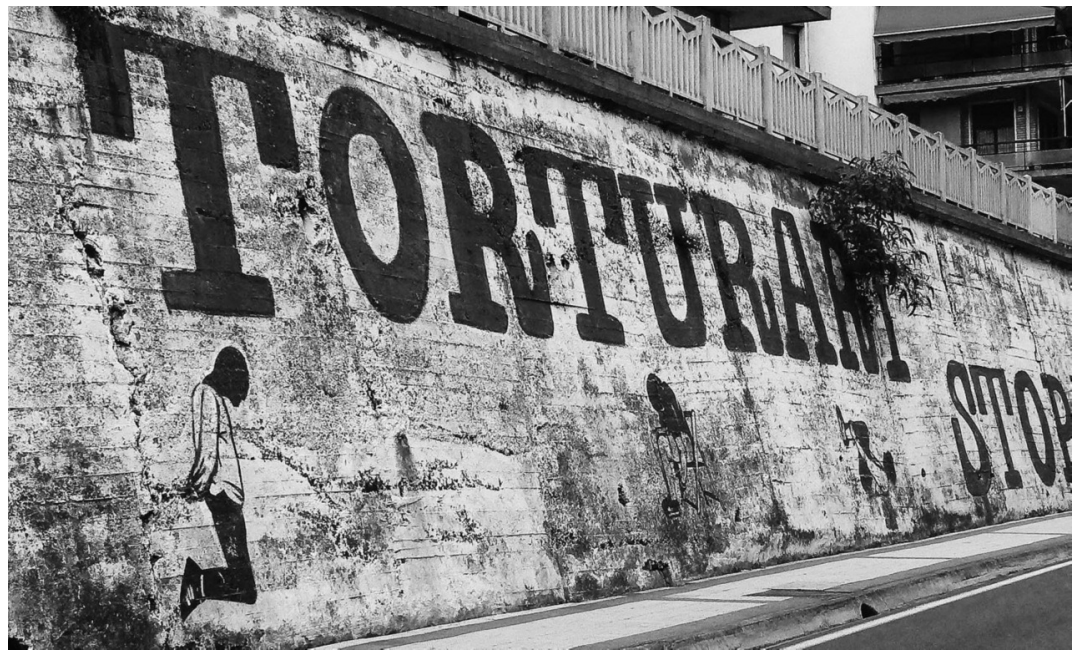

Document 2

Le troisième registre vient rappeler, sous la forme de slogans de soutien, l'activisme de l'ETA dans la région. Cette promotion de la lutte armée s'exprime essentiellement à travers le traçage rapide des trois lettres acronymes de l'organisation paramilitaire souvent précédé d'un «gora» («vive »). Le sigle ETA, comme parfois la représentation figurée d'un militant cagoulé ou le symbole de la hache et du serpent, rappelle la présence encore vivace d'un collectif combattant en dépit d'une répression policière et judiciaire intense, l'inscription dans le quotidien des individus de la surveillance communautaire comme le refus de laisser aux «forces coloniales » la mainmise de l'espace public basque ( $c f$. document 3 ).

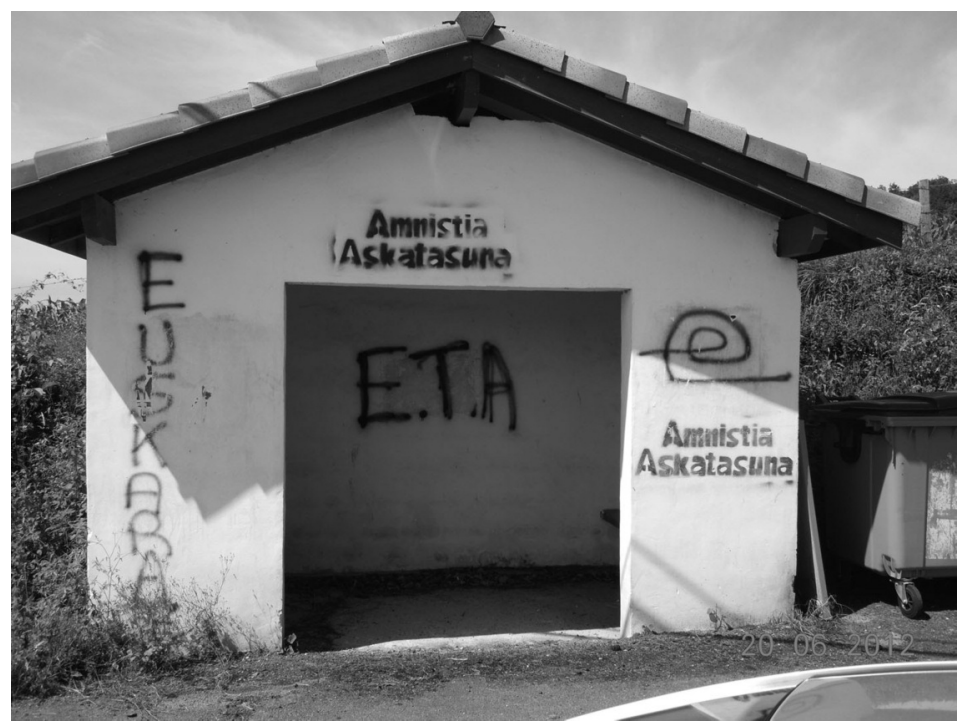

Document 3 
Cette présence graphique de la communauté abertzale dans l'espace public donnera lieu à une réaction très vive du gouvernement socialiste de la communauté autonome basque qui chasse les nationalistes modérés en mai 2009 et initie rapidement une politique d'invisibilisation des traces de cette affirmation contestataire ${ }^{14}$. La presse parlera d'une " guerre des symboles » opposant le gouvernement de Patxi Lopez (PSOE), « refusant la fausse normalité qui règne au Pays Basque, privé d'un espace public citoyen ", aux activistes nationalistes ${ }^{15}$. Aidée par un accord entre le gouvernement basque et certaines institutions comme l'Université du Pays Basque et par la mobilisation d'un appareil législatif national ${ }^{16}$, la police autonome basque (l'Ertzaintza) procédera à plus de 3500 interventions, encadrant les services municipaux, pour effacer les fresques et symboles du nationalisme radical, aboutissant rapidement, selon les termes du ministre de l'Intérieur basque Rodolfo Ares ${ }^{17}$, à une politique de «tolérance zéro » vis-à-vis de l'esthétique abertzale 18 .

Le relevé systématique des occurrences iconographiques en Euskadi nord (Iparralde), permet d'affiner la présentation du corpus iconique qui, s'il présente certains éléments de ressemblance avec le nationalisme du sud, s'en distingue par une moindre radicalité, par l'ancrage autour d'enjeux environnementaux et économiques locaux ainsi que par une mixité prononcée alliant un registre altermondialiste et anticapitaliste.

\section{Outils}

À l'image de ce que l'on retrouve au sud, l'iconographie abertzale au Pays Basque français repose sur différents supports, aux effets visuels variés. Si les dessins et fresques à forte visibilité sont très peu présents, se différenciant ainsi fortement de l'iconographie nationaliste en Irlande du Nord, les graffitis mais surtout les stickers et les pochoirs constituent les relais les plus usités des propagandistes nationalistes, perdant en visibilité mais nettement plus présents dans les lieux de passage (rues des villes). À l'inverse de la Corse où la présence

14. Si le gouvernement basque durcit sa position envers la mouvance abertzale à partir de 2009, la tension autour de la «symbolique ETA » existait déjà avant. De nombreux procès ont opposé le gouvernement autonome ou l'État espagnol aux mairies abertzale sur l'usage du drapeau ou l'appellation choisie pour certaines rues et places. De leur côté les nationalistes radicaux usaient de mises en scène (pantins, mannequins, banderoles, musiques...) affichant leur soutien aux prisonniers et rejetant le pouvoir central « oppresseur».

15. Thibaud C., «La guerre des symboles au Pays Basque », L'Express, novembre 2009.

16. La ley de reconocimiento y reparacion de victimas del terrorismo.

17. Le ministre quittera ses fonctions en août 2012 alors qu'un nouveau gouvernement à majorité nationaliste est en train de se constituer (novembre 2012)

18. On notera ici la politique très différente initiée en Irlande du Nord au sujet des fresques murales et de la visibilité publique des paramilitaires puisque depuis 1994, le gouvernement local a encouragé, non pas l'effacement des murals, mais l'inscription de fresques de réconciliation entre les deux communautés républicaine et loyaliste : Hill A. et White A., "Painting peace. Murals and the Northern Ireland peace project", Irish Political Studies, 27-1, février 2012. 
militante nationaliste s'observe à travers des graffitis ne requérant qu'une faible organisation, la contestation abertzale iconographique est fortement appuyée par des relais organisationnels, aptes à produire affiches, pochoirs ou stickers à des fins de visibilité publique. S'il est difficile de connaitre l'identité des activistes graffeurs, on peut penser que certains groupes très organisés à l'image des Démos, de Segi ou d'Askatasuna ${ }^{19}$ possèdent leurs propres troupes de graffeurs et peintres, agissant au Pays basque.

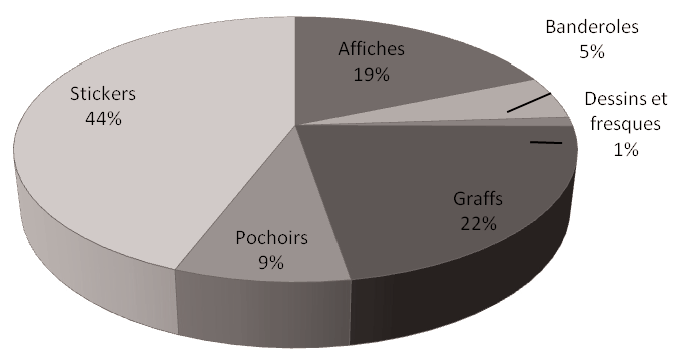

Figure 1 - Répartition des outils iconographiques

On constate une très forte lisibilité des traces iconographiques dans la région basque française. Ce constat tient pour une grande part à la période de réalisation de l'étude, peu de temps après les élections législatives de 2012, véritable séisme local 20 , auquel se rajoute une actualité issue du sud - l'abandon de la lutte armée de l'ETA et l'annonce d'un processus de paix au Pays Basque - qui anime certaines zones militantes du Pays Basque nord. En sus de la lisibilité des traces iconographiques, on peut faire le constat d'une recherche de visibilité politique forte. La plupart des graffitis, affiches ou dessins sont en bord de routes nationales ou des départementales, à la sortie des villes, sur les lieux de passage routier ou dans les centres villes, comme à Bayonne. Les ronds-points ou intersections sont souvent privilégiés. Enfin, dans un même souci de visibilité, l'usage de la couleur, plus attractif que le noir et blanc, est dominant, essentiellement en raison de la forte présence des stickers mais aussi par l'usage instrumental qui est fait des couleurs de l'Ikurrina (le rouge, le vert et le blanc).

19. Démos est une organisation se proclamant comme « le bras non armé du peuple basque » et usant d'un répertoire d'action provocateur et humoristique pour défendre la « démocratie au Pays basque » qui, après dix ans d'activité, s'auto-dissoudra en 2010. Segi est le mouvement de la jeunesse nationaliste radicale et Askatasuna est une organisation, née en 2001 réunissant deux mouvements, transfrontaliers, de soutien aux prisonniers basques.

20. Les législatives de 2012 verront la chute de deux grandes figures de la politique locale, en poste depuis plus d'une décennie : Jean Grenet à Bayonne et Michèle Alliot-Marie à SaintJean-de-Luz. 


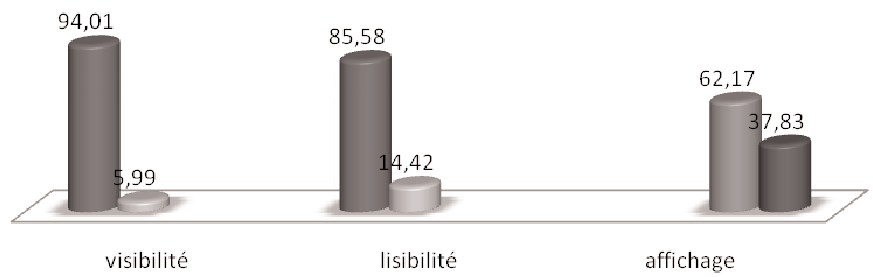

Figure 2 - Qualité de l'affichage iconographique

\section{Thématiques}

Il ressort du classement thématique des catégories de message que véhiculent les traces iconographiques une vraie pluralité des items politiques, parfois très nettement distincte de la contestation nationaliste en Irlande du Nord ou en Corse. Outre l'absence d'affichage raciste et xénophobe, souvent présent sur d'autres terrains de lutte ${ }^{21}$, on constate, à l'inverse de ce qui se passe en Euskadi sud ou en Irlande du Nord, une relative faiblesse de l'affichage violent. Les sigles ETA ou IK 22 ne représentent que $6 \%$ du total des traces iconographiques. Si la période de pacification en cours au Pays Basque explique partiellement cette singularité, on peut aussi penser que la violence politique en Ipparralde n'est pas perçue comme folklorique, à l'image de ce qu'elle peut paraître en Corse, ou partie à l'identité locale, mais comme une vraie menace inquiétante et dérangeante alors que le sud du Pays Basque est confronté depuis près de 50 ans à une violence politique responsable de près de 2500 morts et blessés. Dès lors, l'affichage d'un soutien à la lutte armée n'est peutêtre ni souhaité ni aisé à mettre en ouvre, comme semble l'attester le fait que plus de la moitié de ces occurrences guerrières se retrouvent dans le Pays Basque intérieur, moins peuplé, où l'inscription sauvage s'avère plus discrète.

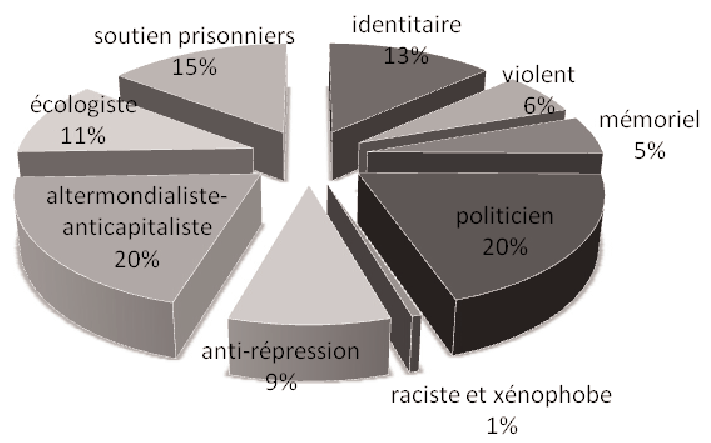

Figure 3 - Rubriques thématiques de la contestation iconographique

21. Voir notre article dans ce numéro consacré à la Corse, pour mieux saisir la différence avec la situation basque.

22. IK est l'acronyme d'Iparretarrak, organisation nationaliste basque clandestine qui sera responsable de plusieurs centaines d'attentats, essentiellement dans le sud-ouest de la France, dans les années 1980-90. 
Dans le même ordre d'idées les références mémorielles ( $c f$. document 4), parfois inscrites en hommage aux militants de la cause, sont assez peu présentes, dans l'espace public de la rue, même si on en trouve d'importantes traces sous la forme d'affiches ou de dessins dans les lieux de sociabilité militante, à l'image des bars ou cafés abertzale du quartier du petit Bayonne.

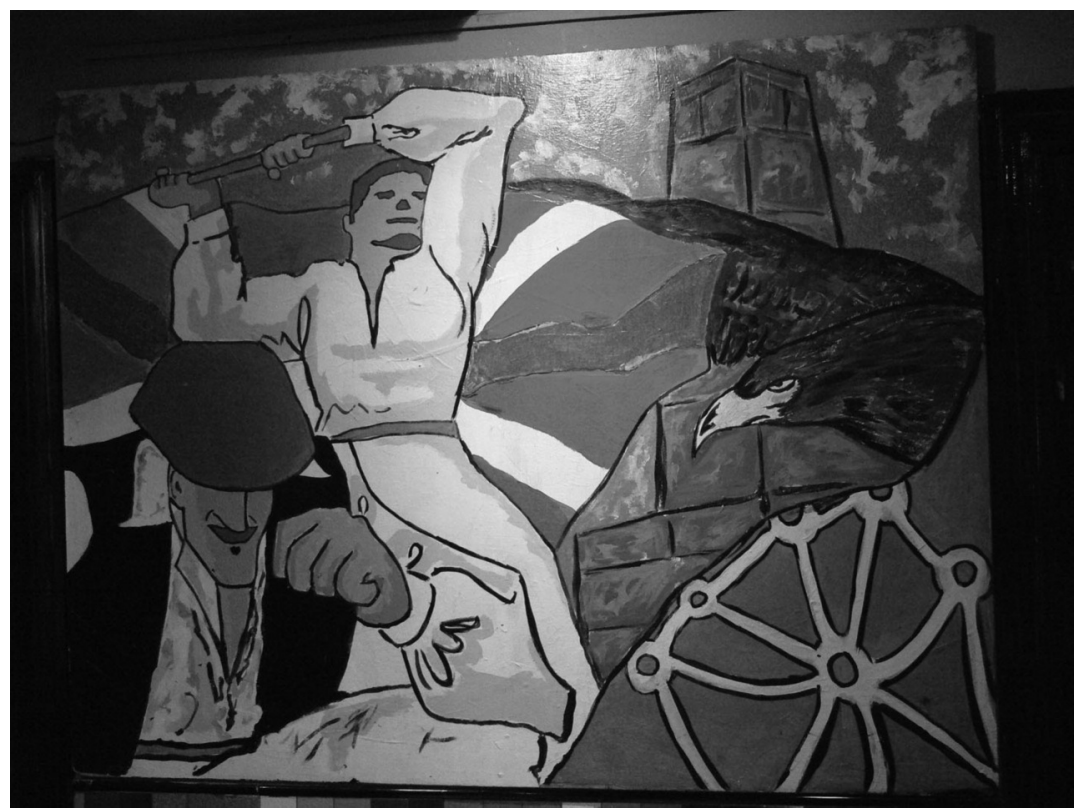

Document 4

À l'inverse, on peut être surpris de la place importante de l'iconographie contestataire de type altermondialiste ou anticapitaliste, très présente sur la côte basque et singulièrement à Bayonne, indiquant l'adoption d'une langue politique d'extrême gauche par les acteurs du nationalisme basque (cf. document 5 ). La répartition croisée des thématiques selon les zones géographiques confirme la spécificité du petit Bayonne puisque presque $87 \%$ des marques iconographiques altermondialistes ou gauchistes s'y concentrent (alors qu'on n'en rencontre aucune dans le Pays Basque intérieur et $13 \%$ sur le reste de la côte).

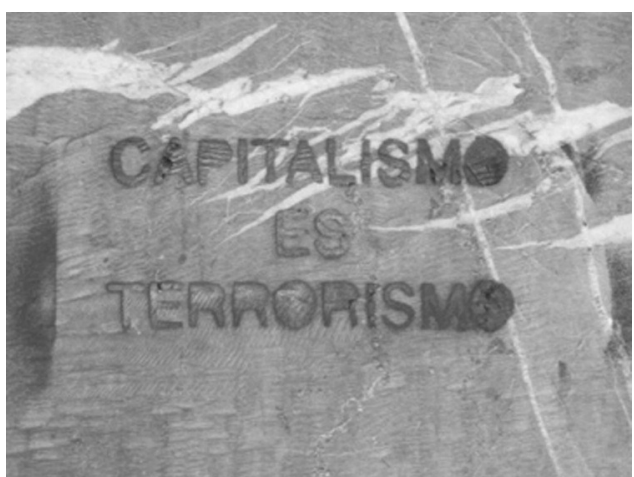

Document 5 
La forte proportion d'affichage de type politicien est due à la période électorale (législatives 2012) à laquelle les abertzale ont pris une part active. Elle résulte aussi de la présence dans l'espace public de structures politiques nationalistes diverses, allant des partis, associations de jeunes (SEGI) aux syndicats (le LAB - Langile Abertzalen Batzordeak - en particulier). Cette répartition est sensiblement égale dans toutes les zones géographiques.

Une rubrique importante, depuis toujours au sein d'un conflit qui a fait près de 1000 morts et plus de 500 prisonniers, concerne le soutien à ces derniers (cf. document 6). Si on additionne les traces iconographiques marquant la défense des prisonniers et réclamant leur regroupement au Pays Basque, avec celles relatives à la contestation de la répression (refus du mandat d'arrêt européen ou condamnation des mauvais traitements subis par les prisonniers basques dans les prisons espagnoles), on obtient le plus gros pourcentage thématique de la contestation iconographique ( $24 \%$, soit un quart du corpus). Ce sont principalement dans les villes et villages où sont présentes les associations de soutien aux presos (prisonniers), que ce type d'iconographie est dominant. Bayonne en constitue sûrement l'épicentre (71\% des marquages antirépression et $62,3 \%$ des marquages pro-prisonniers). Notons ici que l'appui aux prisonniers, s'il est intimement lié à la présence de l'ETA, se distingue cependant du soutien direct à l'organisation clandestine basque : la défense des prisonniers est partagée au-delà des cercles abertzale et semble souvent relever de convictions humanitaires ou de solidarités familiales, quand l'appel à l'ETA implique nettement plus la seule branche la plus radicale du mouvement abertzale.

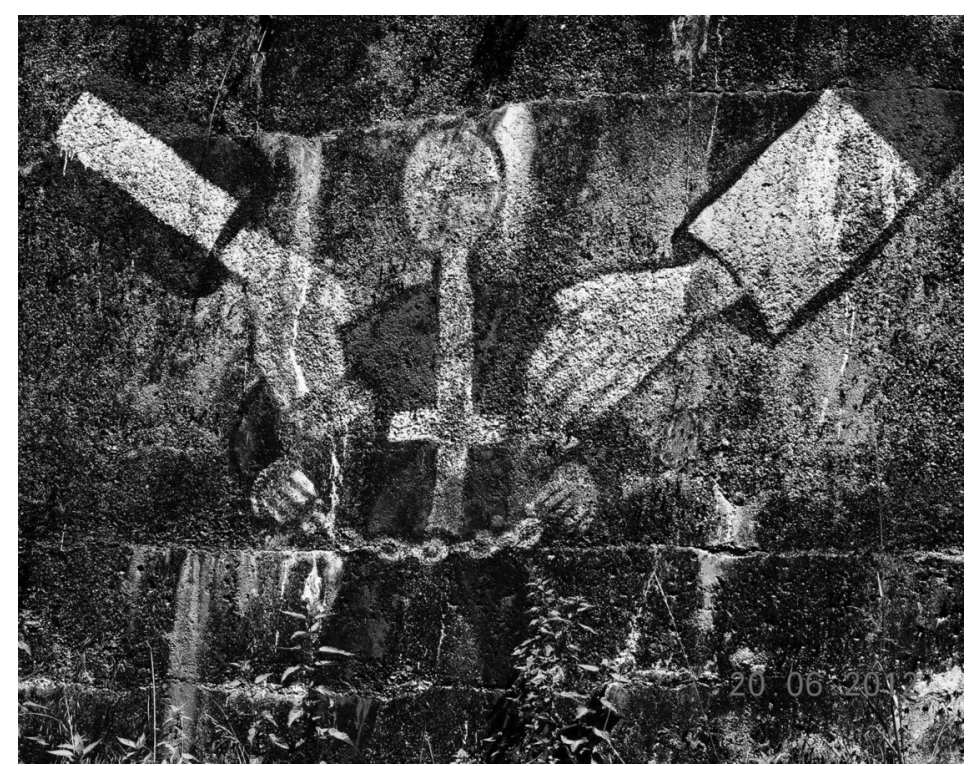

Document 6 
Deux dernières rubriques, étroitement liées, retiennent l'attention. La première, relative à la contestation écologiste, est principalement animée d'un point de vue partisan par les formations abertzale : la majorité du corpus iconographique de type écologiste porte sur le refus du traçage de la nouvelle ligne TGV. En cela, elle rejoint la thématique identitaire qui, pour une bonne part, porte sur la lutte contre la spéculation foncière et l'installation sur la terre basque de résidences secondaires, responsables d'une augmentation impressionnante du prix du foncier ( $c f$. document 7 ). Ces deux thématiques - unies par leur projet de défense de l'identité foncière et territoriale - regroupent elles aussi un quart du corpus. Sans surprise, on retrouve le marquage écologiste anti-TGV sur la côte atlantique, lieu de passage programmé de la ligne à grande vitesse (72 \% des marquages écologistes), alors que le marquage identitaire anti-spéculation est nettement plus distribué, attestant la montée des prix du foncier sur l'ensemble du territoire basque (40\% sur la côte et $60 \%$ dans l'intérieur). En proposant un registre d'explication liant spéculation immobilière, atteinte à l'environnement et fuite contrainte des locaux, la contestation iconographique de type écologiste s'ancre pleinement dans les thématiques des mouvements nationalistes.

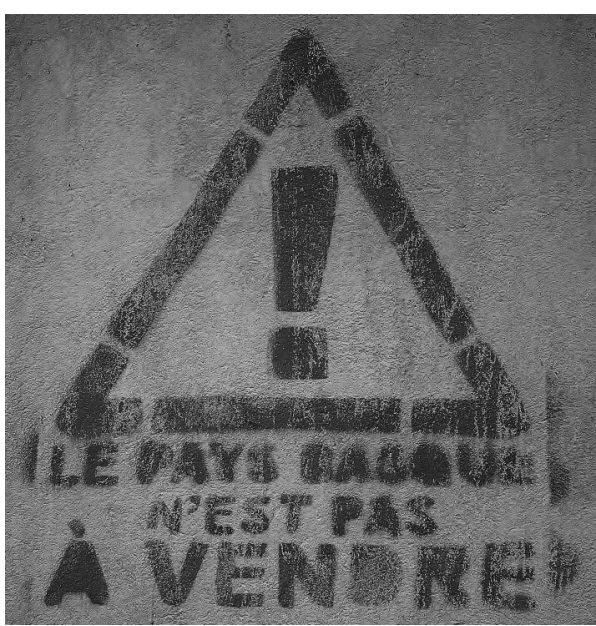

Document 7

On notera avec une certaine surprise l'absence quasi-totale de références identitaires de type historique à l'image de la couverture iconographique nationaliste en Ulster. Si la totalité des tags et mots d'ordre abertzale sont rédigés en euskera, on ne trouve guère d'autres signes de la lutte identitaire ${ }^{23}$.

Le tableau ci-dessous permet de mieux mesurer la répartition thématique des traces iconographiques en fonction des trois principales zones dans lesquelles la contestation opère : Bayonne, centre névralgique de la contestation abertzale fondue dans un registre altermondialiste devenu dominant; la côte atlantique, plus centrée sur des préoccupations environnementales liées au tracé d'une ligne à grande vitesse, et le Pays Basque intérieur, lieu d'expression d'un nationalisme historique autour des grandes figures (ETA) et revendications du mouvement (défense de la langue, soutien aux prisonniers).

23. À l'inverse de ce qu'affirmait Lymann Chaffee, percevant dans les années 1990 le street art politique nationaliste comme un mode d'imposition identitaire face à la présence du modèle castillan in Chaffee L., Political Protest and Street Art. Popular Tools for Democratization in Hispanic Countries, London, Greenwood Press, 1993, p. 69. 


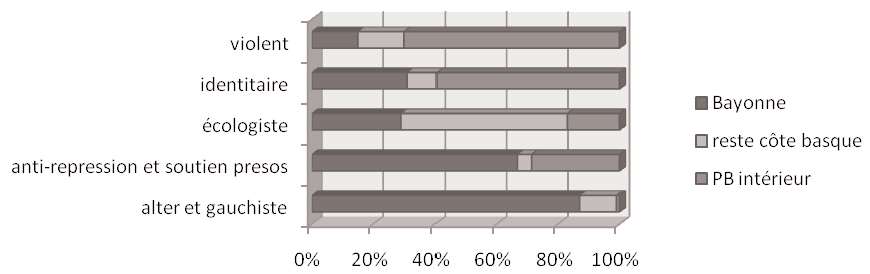

Figure 4 - Répartition thématique des traces iconographiques selon les zones géographiques

À l'inverse de ce qui est perceptible en Corse ou en Irlande du Nord, le relevé a également montré une très forte présence en Euskadi nord des tags, fresques et graffs non politiques, s'inscrivant dans une culture périurbaine de type hip hop, essentiellement concentrée dans les grandes villes de la côte basque et probablement fortement influencée par la présence d'une sociabilité ludique mais aussi commerciale issue du monde du surf. Sans prétendre à un comptage exhaustif, il apparaît que les abertzale n'ont pas le monopole de la présence iconographique murale et sont concurrencés par un registre du street art à dimension sportive ou proprement artistique, isolant dans certaines zones (la côte par exemple), un message nationaliste nettement moins visible et audible :
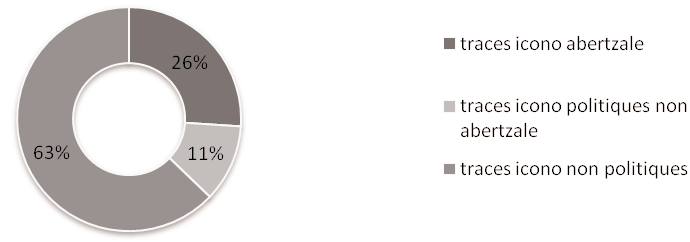

Figure 5 - Répartition des types de traces iconographiques

\section{Localisation}

Si le combat nationaliste s'affiche sur l'ensemble du Pays Basque, il est inégalement réparti selon les zones géographiques, opposant un univers rural intérieur moins marqué par les représentations nationalistes à un monde urbain, principalement situé en bord de mer et nettement plus soumis à une propagande partisane abertzale. Le schéma suivant illustre cette dichotomie :

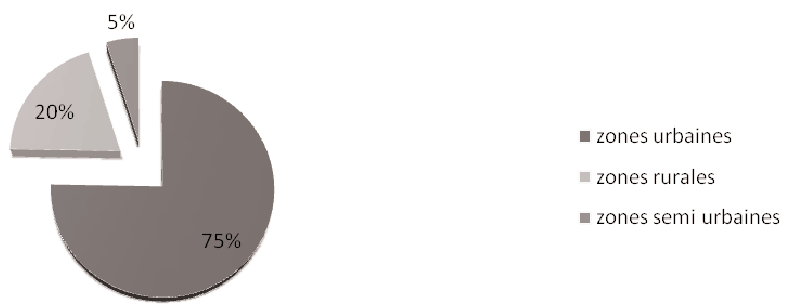

Figure 6 - Répartition des traces iconographiques selon les zones géographiques 
Pourtant cette répartition demeure trompeuse à deux égards. C'est tout d'abord la place centrale de Bayonne qui retient l'attention, et singulièrement au sein de cette ville le quartier du petit Bayonne qui constitue l'épicentre de la contestation nationaliste en Pays Basque nord. Presque la moitié du corpus provient de cette seule ville et plus de $90 \%$ du corpus bayonnais est concentré dans le « petit Bayonne » historique, marquant ce quartier comme le principal espace de sociabilité militante abertzale en Ipparalde. Par contre, si Bayonne demeure le cour du nationalisme basque, la contestation y est plus ouverte qu'ailleurs puisque $68 \%$ des stickers et graffs relevés peuvent être considérés comme non strictement abertzale, débordant sur une contestation anarchiste, antifasciste et altermondialiste. Au contraire, dans le Pays Basque rural, la contestation est à plus de $95 \%$ strictement nationaliste sans référence explicite à d'autres thématiques et souvent directement associée à un soutien aux formations les plus extrémistes du mouvement basque.

Autre élément d'interrogation, la côte atlantique basque (de Hendaye à Biarritz) qui concentre les trois quarts de la population du Pays Basque est extrêmement pauvre en iconographies abertzale. Les raisons sont multiples : espaces urbains et périurbains riches, au foncier très élevé et fortement ancrés à droite, ces villes côtières sont sociologiquement peu réceptives à la problématique abertzale. On peut estimer également que les acteurs nationalistes y sont peu présents compte tenu des prix de l'immobilier, poussant les locaux dans les zones intérieures ou dans certains quartiers préservés de la fièvre spéculative du bord de mer. Enfin, vitrine touristique de la région, la côte basque bénéficie d'une politique d'équipement efficace et active comme l'atteste l'importance des traces d'effacement perceptibles dans des villes comme SaintJean-de-Luz ou Biarritz. On ajoutera que la domination de la culture «surf » sur la jeunesse locale et le brassage des populations attirées par ce sport laisse peu de place - au sens physique du terme - aux inscriptions abertzale.

L'intérieur rural du Pays Basque affiche une présence importante de graffs et tags abertzale puisque dans la région de Saint-Jean Pied-de-Port / SaintÉtienne-de-Baigorry, ce sont plus de $17 \%$ des inscriptions nationalistes qui sont recensées. Les trois autres régions intérieures (Cambo-les-Bains, La Bastide-Clairence et Mauléon / Saint-Palais) sont moins bien fournies mais contiennent le long des routes nationales et départementales moins passantes, d'imposantes marques iconographiques (fresques ou tags d'envergure à forte visibilité), nettement moins fréquentes sur la côte :

\begin{tabular}{|l|c|c|c|c|c|c|}
\hline $\begin{array}{l}\text { Zones } \\
\text { géographiques }\end{array}$ & Bayonne & Côte basque & $\begin{array}{c}\text { Saint-Jean } \\
\text { Pied-de- } \\
\text { Port }\end{array}$ & $\begin{array}{c}\text { Cambo-les- } \\
\text { Bains }\end{array}$ & $\begin{array}{c}\text { La Bastide- } \\
\text { Clairence }\end{array}$ & $\begin{array}{c}\text { Mauléon / } \\
\text { Saint-Palais }\end{array}$ \\
\hline $\begin{array}{l}\text { \% de traces } \\
\text { iconographiques }\end{array}$ & 49,5 & 13 & 17,42 & 6,4 & 6,4 & 6,2 \\
\hline
\end{tabular}

Figure 7 - Répartition des traces iconographiques abertzale selon les zones géographiques 
Si on s'intéresse à la localisation par supports des traces iconographiques, on constate une variété attendue des moyens d'affichage des messages politiques :

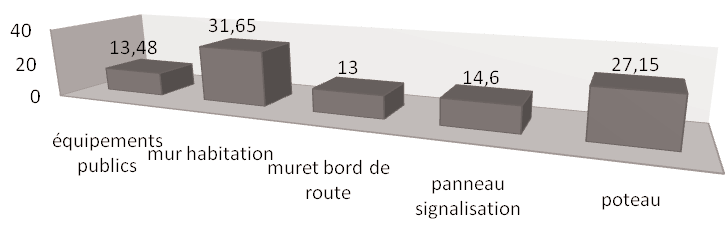

Figure 8 - Répartition par support (en \%)

La répartition par supports est trompeuse. Si elle fait apparaître statistiquement une écriture ou un affichage sauvage sur les murs des habitations privatives, cela est essentiellement dû à la domination iconographique dans le petit Bayonne où seuls des façades d'immeubles peuvent servir de supports naturels. Ailleurs, l'observation témoigne d'une très faible occupation des murs blancs des maisons traditionnelles basques, pourtant excellents supports possibles d'écriture. C'est le plus souvent sur les maisons abandonnées et décrépies que fleurissent les écritures sauvages. Outre la difficulté à taguer un mur de maison individuelle et l'effacement systématique par les propriétaires des tags salissants, on peut émettre l'hypothèse que l'architecture typique de maisons basques, signe d'une identité « nationale » recherchée et valorisée ${ }^{24}$, suffit à marquer le territoire de ce banal nationalism dont parlait Michael Billig ${ }^{25}$. L'empreinte abertzale pourrait être pensée ici comme redondante, en plus d'être dégradante.

Sans surprise c'est donc sur les équipements publics (relais EDF, poubelles, façades administratives) et poteaux électriques que sont présentes les écritures sauvages dans une entreprise d'occupation de l'espace public à domination étatique. Le faible nombre de panneaux de signalisation taggués ainsi que l'absence d'effacement des indications en français au profit d'une affirmation de l'euskera surprennent plus pour qui connaît le marquage nationaliste en Corse. Ce refus du roadsign protest 26 est difficilement explicable sauf à penser que l'identité basque est suffisamment inscrite dans l'espace paysager,

24. Sur le lien entre identité et architecture, on se reportera à Braud P., Etes-vous catholique... ?, Paris, Presses de Science po, 1998, pp. 107 et s., et à De Biase A. et Rossi C. (eds.), Chez nous : Identité et territoire dans les mondes contemporains, Paris, Éditions de la Villette, 2006. Voir également dans le cas américain, Vale L., Architecture, Power and National Identity, New Haven, Yale University Press, 1992.

25. Billig M., Banal Nationalism, Londres, Sage, 1995.

26. Jeremy Mac Clancy évoque au milieu des années 2000 cette pratique du maquillage des panneaux rédigés en castillan par des militants de la défense de l'euskera: Expressing Identities in the Basque Arena, Oxford, SAR Press, 2007, p. 157 et s. 
dans les pratiques quotidiennes des habitants et institutionnellement soutenue, pour ne pas nécessiter une opposition frontale avec les signes extérieurs de la présence d'État.

\section{Logiques de cadrage et rationalité pratique de l'iconographie abertzale}

Les murs bavards du Pays Basque offrent finalement d'intéressants cadres de lecture et d'analyse de la situation politique dans la région, non qu'ils exhibent une donnée sociale indiscutée, mais plutôt des récits singuliers de transfiguration de la réalité politique dans lesquels la lutte nationaliste se présente comme une alternative morale face à un État aux agissements pensés comme immoraux ${ }^{27}$. Ces «cadrages picturaux » singuliers fonctionnent comme d'efficaces « dispositifs de sensibilisation" ${ }^{28}$, destinés à agir sur les affects des spectateurs et à susciter des émotions variées qui favorisent la prise de conscience politique ou l'activisme militant.

Derrière ces récits mis en image, à forte valeur émotionnelle, transparait un intérêt stratégique à l'usage de l'iconographie, permettant le dire en public, l'agir collectif et la représentation communautaire.

\section{Cadrage et dispositifs de sensibilisation}

Comme tous les entrepreneurs de mobilisation, les formations et acteurs nationalistes s'efforcent d'influer sur les représentations du réel à travers la construction de «cadres d'action collective ", véritables ensembles de croyances et représentations destinés à pousser à l'action en mettant en scène, soit l'injustice ou l'amoralité flagrante d'une situation, soit l'inscription identitaire bafouée ou censurée. En réintroduisant dans l'analyse de l'action collective cette question des croyances et des interprétations, certains théoriciens comme Snow, Benford ou Oberschall vont insister sur l'importance des représentations de la situation pour comprendre l'émergence d'une lutte sociale et politique qui ne saurait se résumer à la simple expression d'une colère ou frustration ou à la mobilisation de ressources rares indispensables pour mener l'action 29. On pourrait avancer ici l'usage de «cadres picturaux » mis en avant par les nationalistes basques pour favoriser un processus de cadrage multiple,

27. On proposera ici une lecture en terme de cadrage cognitif de l'action contestataire iconographique. Voir à ce sujet Snow D., «Analyse des cadres et mouvements sociaux » in Cefaï D. et Trom D. (eds.), Les formes de l'action collective, Paris, éd. EHESS, 2002, p. 35. Voir également Contamin J-G., «Cadrages et luttes de sens » in Fillieule O., Agrikoliansky E. et Sommier I. (eds.), Penser les mouvements sociaux, Paris, La Découverte, 2010, pp. 55 et s.

28. Siméant J. et Traïni C., "Pourquoi et comment sensibiliser à la cause ?» in Traïni C. (ed.), Émotions... Mobilisation!, Paris, Presses de science po, 2009, p. 13

29. Snow D. et Benford R., "Ideology, frame resonance and participant mobilization" in Klandermans B., Kriesi H. et Tarrow S. (eds.), From Structure to Action: Comparing Social Movement Research across Cultures, Greenwich, JAI Press, 1988 ; Oberschall A., "The manipulation of ethnicity: from ethnic cooperation to violence and war in Yugoslavia”, Ethnic and Racial Studies, 23-6, novembre 2000. 
nettement plus direct qu'il est visuellement très présent sur les murs des villes et très attractif eu égard à la force des slogans et au poids des symboles mobilisés 30 .

Fondée sur quelques «cadres cardinaux » (master frames) de portée générale et universellement adoptables (de types écologistes ou moraux, en appelant au rejet de la torture et à la défense de notre environnement commun), l'entreprise de framing pictural des nationalistes basques repose sur une lecture plurielle de la situation. Travail de connexion des cadres d'expérience (frame bridging) lorsqu'il s'agira de fusionner les luttes étrangères (Irlande du Nord ou modèle palestinien par exemple) au cas domestique ou de fondre l'activisme anticapitaliste dans la défense d'une singularité locale. Travail d'amplification (frame amplification) en opérant une désingularisation du cas basque pour porter la revendication nationaliste sur un discours plus général de respect des identités ou de défense de la terre. Travail d'extension du cadre (frame extension) également en débordant sur le terrain de la lutte écologique ${ }^{31}$ ou de la lutte anti-spéculative ${ }^{32}$, favorisant ainsi une adhésion du plus grand nombre aux thématiques abertzale. Enfin, travail de transformation du cadre de la lutte (frame transformation) à travers lequel l'affrontement ethnonationaliste devient progressivement un des éléments du combat altermondialiste et anticapitaliste, incluant dans le registre des valeurs abertzale une thématique plus large mais aussi plus facile à valoriser au sein d'un espace public agrandi.

L'efficacité de cette entreprise de cadrage au Pays Basque repose sur l'usage d'une iconographie en résonance avec le terreau socio-culturel local en ce qui concerne un certain nombre de traits du framing : le catholicisme rural propre à la région favorise la mise en lumière d'appels à la défense de l'environnement ou la sensibilité collective aux thèmes de la protection de la communauté et de ses membres face à la menace policière extérieure. La situation géographique du Pays Basque, à cheval entre deux grandes nations, valorise les appels à la sauvegarde de l'identité communautaire, tout comme le souvenir d'une Espagne longtemps dictatoriale aux portes de l'Euskadi sert les ambitions démonstratives d'une police violente et d'une répression opaque. Le faible nombre de sigles ouvertement marxisants ou d'appels explicites à la lutte armée (moins de $6 \%$ ) témoigne du souci des entrepreneurs nationalistes de conserver un cadrage fidèle à l'esprit de l'époque, largement méfiant vis-àvis des utopies révolutionnaires, et soucieux des légitimes aspirations à la quiétude et à la paix.

30. Dans le même ordre d'idée sur l'usage des arts graphiques pour favoriser l'activisme chez les militants d'Act up, voir Reed T.V., The Art of Protest: Culture and Activism from the Civil Rights Movement to the Streets of Seattle, Minneapolis, University of Minnesota Press, 2005.

31. Le refus de la ligne grande vitesse TGV est très présent sur les murs de la côte basque.

32. L'importance numérique de l'équation « $35864=$ Minbizi $»(35864=$ cancer $)$ sur les murs basques se réfère au nombre de résidences secondaires présentes dans la région et responsable, selon les nationalistes, de la hausse du foncier depuis quelques années. 
L'iconographie rebelle qui orne les murs du Pays basque constitue donc un filtre culturel de la réalité sociale et politique en Euskadi. Elle participe à l'érection de véritables «paysages de sens ", "compris spontanément par ceux qui s'y meuvent quotidiennement ou qui doivent être déchiffrés par ceux qui (leur) sont étrangers » 33 . Ces formes iconographiques disponibles publiquement fonctionnent à la fois comme des aspirations à bousculer l'ordre dominant des qualifications et des frontières de classement ainsi que comme des récits en propre. Mots d'ordre de contestation de la légitimité dominante, les marques iconographiques, le plus souvent rédigées en euskera, critiquent les dispositifs officiels de catégorisation et de classement - à commencer par l'usage de la langue officielle - affirmant haut et fort, à rebours de la politique institutionnelle, que «le Pays basque n'est pas la France », que « l'État est terroriste » ou que "vive ETA ». Récits collectifs proposés à la vue de tous, les traces iconographiques abertzale racontent une autre histoire que celle publiquement enseignée, une histoire de lutte dominée par la figure de l'ETA, organisation protectrice pour «son peuple » et menaçante pour ses ennemis, une histoire de résistance face à une Espagne assassine et des pouvoirs financiers prédateurs, une histoire de protection d'une nature belle mais inquiétée par des appétits externes agressifs, une histoire d'identité fragilisée mais puissamment investie par toute une population ${ }^{34}$.

Cadrage et storytelling au cœur des représentations iconographiques participent à la mise en place des dispositifs de sensibilisation initiés par les acteurs nationalistes « afin de susciter des réactions affectives qui prédisposent ceux qui les éprouvent à s'engager ou à soutenir la cause défendue » ${ }^{35}$. En proposant au spectateur comme au militant ou au sympathisant des symboles, des sigles, des formules, des cartes, des dessins, des slogans, des portraits ou des couleurs, les graffs abertzale visent à susciter des registres émotionnels variés, à la fois positifs et négatifs, débouchant sur l'activation d'affects et de sentiments, dont on peut penser qu'ils sont aussi à la source de l'activisme comme de la représentation communautaire du groupe ${ }^{36}$. La trace iconographique, souvent répétée et largement visible, provoque une réaction émotionnelle, immédiate et instinctive, qui peut être négative ou positive. Elle encourage aussi parfois un marquage " affectuel » plus largement durable qui favorisera, s'il est suivi d'un efficace monitoring des organisations nationalistes, une vision plus pérenne d'un collectif uni sur la base de l'identité partagée ou de la peur suscitée. On proposera de résumer comme suit les dispositifs de sensibi-

33. Cefaï D., Pourquoi se mobilise-t-on? Les théories de l'action collective, Paris, La Découverte, 2007, p. 483. Sur le concept de paysage de sens, voir Zerubavel E., Social Mindscapes. An invitation to cognitive sociology, Harvard, Harvard University Press, 1999.

34. Sur l'importance des récits dans les mouvements sociaux, voir Polletta F., It was like a fever: Storytelling in protest and Politics, Chicago, Chicago University Press, 2006.

35. Siméant J. et Traïni C., op. cit., p. 13.

36. Sur le rôle des affects et des émotions dans l'action collective, Sommier I., «Les états affectifs ou la dimension affectuelle des mouvements sociaux » in Fillieule O. et al. (eds.), op. cit., pp. 185 et s. Voir également Jasper J., " Du symbole à l'émotion » in Sommier I. et Crettiez X. (eds.), Les dimensions émotionnelles du politique, Rennes, PUR, 2012. 
lisations iconographiques et leurs effets supposés, sans présumer ici des intentions stratégiques des acteurs anonymes dont on peut malgré tout penser que leur investissement graphique risqué n'est pas sans visées instrumentales ${ }^{37}$ :

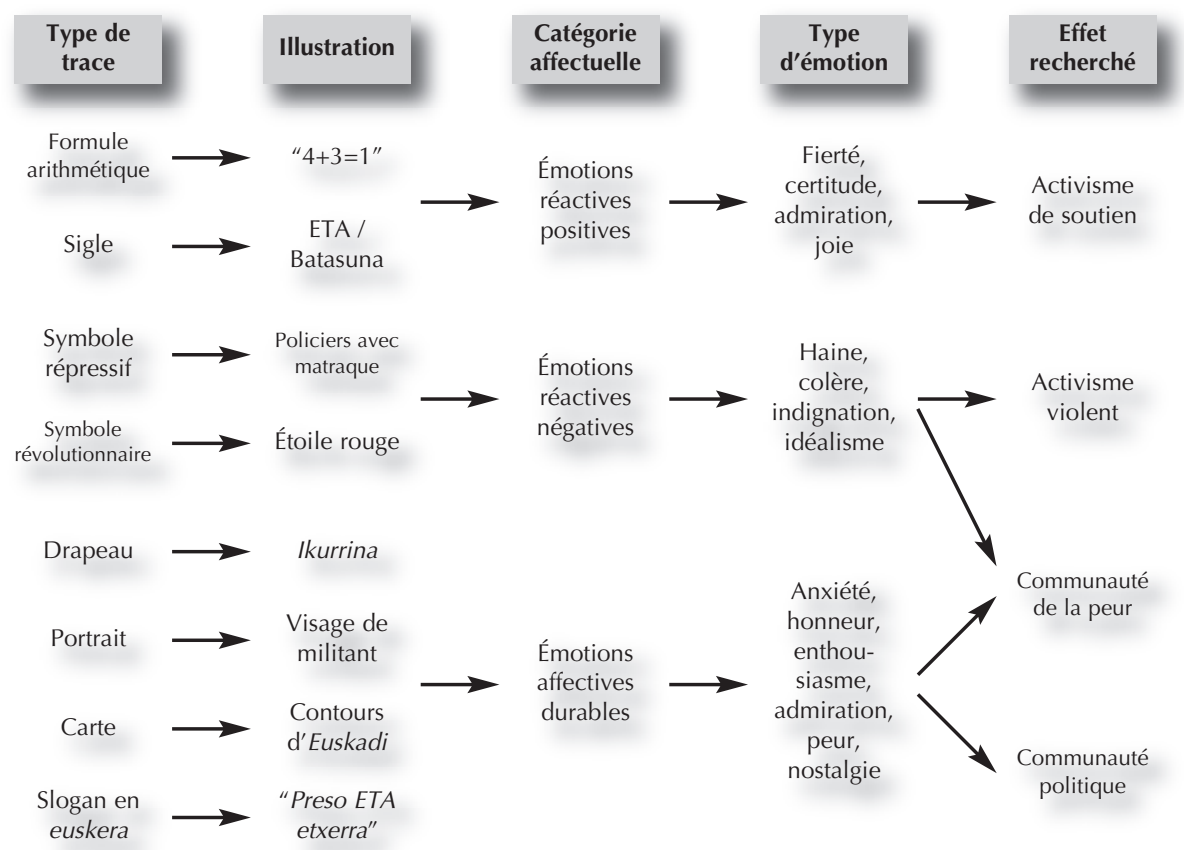

Figure 9 - Les dispositifs de sensibilisation iconographiques

\section{La rationalité pratique de l'iconographie}

En pensant les dispositifs de sensibilisation comme des mécanismes stratégiquement mis en place pour susciter de l'adhésion et de l'engagement, on souligne la part d'instrumentalisation des techniques iconographiques chez des entrepreneurs de cause que sont les organisations et militants abertzale ${ }^{38}$. On distinguera trois usages généraux et variés de l'iconographie politique contestataire : dire, agir et représenter ${ }^{39}$.

37. L'équation « $4+3=1$ », très répandue au Pays Basque, revient à penser l'unité d'Euskal Herria réunissant toutes ses provinces (les 3 régions basques françaises et les 4 provinces espagnoles dont la Navarre). On retrouve un semblable usage de l'arithmétique dans d'autres luttes comme en Palestine où les murs des territoires occupés affichent " $1948+1967$ = all Palestine ", comme si l'usage de la rigueur mathématique valait certification de vérité politique in Peteet J., "The writing on the walls: The graffiti of the Intifada", Cultural Anthropology, 11-2, 1996, p. 149.

38. Sur cette dimension instrumentale des dispositifs de sensibilisation appliqués à la cause animale, voir Traïni C., La cause animale. Essai de sociologie historique, Paris, PUF, 2010.

39. Chaffee L., Political Protest and Street Art. Popular Tools for Democratization in Hispanic Countries, London, Greenwood Press, 1993. 
Dire : l'iconographie contestataire peut s'apparenter à un low technology medium, particulièrement utile à ceux qui ne bénéficient pas naturellement d'un accès privilégié à l'espace médiatique. Véritable caisse de résonance communautaire, elle permet d'exister au côté ou en opposition aux grands média locaux et nationaux, participant à la mise sur agenda de thématiques faiblement publicisées (la question des prisonniers basques par exemple). L'inscription de thématiques identitaires sur les murs basques permet également de forcer le regard médiatique officiel et d'attirer l'œil des journalistes, usant pour se faire de l'anglais comme langue de communication ${ }^{40}$. Au-delà de cette fonction de connexion médiatique, l'iconographie abertzale sert aussi l'annonce militante en usant des murs comme des relais informationnels à destination des sympathisants de la cause. La tenue d'un événement important (concert, conférence de soutien aux prisonniers, manifestations) sera annoncée sur les murs, constituant l'espace public de la rue en caisse de résonance effective d'une actualité militante surprésente. Il en va de même de la tenue de meetings électoraux ou de l'existence des partis abertzale qui trouvent sur les façades les moyens de leur propagande (plus de $20 \%$ des inscriptions iconographiques en Iparralde). Enfin, la parole subversive abertzale peut aussi s'afficher sur les murs, participant au monitoring nationaliste de la société basque et à l'inscription d'une morale d'opposition à la pratique citoyenne, refusant la délation ou contestant le soutien aux institutions ${ }^{41}$.

Agir : l'iconographie n'est pas que parole; elle est aussi action quand elle met en place les conditions de l'activisme militant en préparant un cadre visuel d'expérience aux sympathisants abertzale ${ }^{42}$. La couverture iconographique du quartier du petit Bayonne, riche et exaltée, mélange d'appels à la révolte, de dénonciation de la répression et de glorification de l'action directe, sert à n'en pas douter la formation d'habitus - et de pratiques - contestataires, comme en témoigne la récurrence des phénomènes manifestants sur cette zone. Haut lieu de la culture activiste basque, Bayonne mais aussi le vieux quartier de Donostia ou Hernani attestent la capacité des abertzale à prendre le contrôle de l'espace de la rue et à marquer de leur empreinte des lieux sociaux, présentés comme libérés de la tutelle étatique. L'iconographie de rue, quand elle est dense et homogène dans sa finalité, favorise le marquage des consciences collectives et vient rivaliser directement avec la puissance publique dans sa prétention à définir la citoyenneté. Enfin, la présence, par-

40. On retrouve dans Bayonne de nombreux pochoirs « Freedom for Basque Country ». Comme le reconnaît Julie Peteet dans le cas de l'usage de l'anglais en Palestine : "Graffiti constitued a voice for those who felt voiceless in the international area », op. cit., p. 145.

41. Avant la politique d'effacement du gouvernement socialiste en 2009 , de nombreux patronymes de policiers ou soutiens aux pouvoirs publics non nationalistes étaient directement inscrits, assortis d'une menace non équivoque, sur les murs des quartiers abertzale de Donostia, Bilbao ou Hernani.

42. Voir à ce sujet Mac Clancy J., "The culture of radical basque nationalism”, Anthropology Today, 4-5, octobre 1988, pp. 17-19. Sur l'importance du cadre de conditionnement à la participation violente, voir Crettiez X., Violence et nationalisme, Paris, Odile Jacob, 2006, pp. 22 et s. 
tout en Euskadi, de traces iconographiques contestataires, vient convaincre l'observateur de l'existence d'un collectif résistant réel et actif, traduisant en actes visibles la lutte clandestine et invisible qui s'y déroule.

Représenter : l'usage dominant de l'euskera dans l'iconographie nationaliste basque participe évidemment à l'exigence militante de survivance de la langue, emblème de la singularité "nationale » et, inscrite sur les murs, marque de l'activisme abertzale. L'iconographie fait donc exister aussi dans ses formes mêmes, une communauté résistante, distincte et rebelle, assise sur un sentiment de peur partagée, largement exprimée dans la dénonciation murale des actes de torture et de la répression subie (plus du quart des traces iconographiques au nord et probablement plus encore au sud). L'unité linguistique qui fonde le sentiment nationaliste basque se double ainsi d'une unité émotionnelle, reposant sur le refus collectif de la menace extérieure et sur l'entretien, à grande force de slogans et d'images parfois difficiles, des souvenirs d'une répression continue. Cette mise en image de la violence des États sert enfin l'ultime objectif de l'iconographie murale abertzale : transcrire le récit d'une opposition historique avec Madrid et Paris, alimentant ainsi l'existence d'une « question basque » agitant l'actualité de ces deux grandes nations.

L'iconographie nationaliste abertzale, bien que plurielle et pas toujours homogène selon le versant de la frontière, repose massivement sur la célébration d'une identité menacée, d'un collectif de prisonniers isolés et fragilisés par une répression présentée comme aveugle ainsi que par le rappel de la présence potentiellement active de l'ETA, figure mythifiée de la lutte nationaliste. Audelà de ces thématiques traditionnelles, les murs basques expriment également des préoccupations environnementales et anti-capitalistes qui fondent la lutte abertzale dans une modernité politique rarement présente sur d'autres terrains de lutte similaires (Irlande du nord ou Corse par exemple). Les slogans mis en image en disent long sur les évolutions et la dynamique de la problématique nationaliste en Euskadi. Ils permettent aussi de mesurer les ambitions mobilisatrices des entrepreneurs militants, usant de l'image et du graff comme autant de dispositifs de sensibilisation favorables à l'activisme et proposant des récits qui complètent ce que la parole publique freine à admettre. L'analyse sociologique gagne à vouloir saisir par le bas et par les marges des ambitions politiques qui ne sont pas toujours exprimées publiquement. Le regard sur les murs n'est pas qu'anecdotique; il exprime durablement et à la vue de tous un discours public mais aussi caché que le seul entretien ne parvient pas toujours à dévoiler. 